\title{
Nanobiology for the Pharmacology of Cellular Ion Channels
}

\author{
Alexander V. Kabanov • Mikhail P. Kirpichnikov • \\ Alexey R. Khokhlov
}

Received: 5 December 2008 /Accepted: 17 December 2008 / Published online: 24 January 2009

(C) Springer Science + Business Media, LLC 2009

\begin{abstract}
Writing this editorial is especially pleasing. First, it provides us an opportunity to introduce new directives to the field of Neuroimmune Pharmacology and to explain why the field of nanomedicine is likely an important part of its future growth and development. Second, it is an opportunity to showcase research in this area currently operative in Russia
\end{abstract}

A. V. Kabanov $(\bowtie)$

Center for Drug Delivery and Nanomedicine,

Department of Pharmaceutical Sciences, College of Pharmacy,

University of Nebraska Medical Center,

985830 Nebraska Medical Center,

Omaha, NE 68198-5830, USA

e-mail: akabanov@unmc.edu

e-mail: akabanov@cox.net

URL: http://cddn.unmc.edu

URL: http://nanomedicine.unmc.edu

A. V. Kabanov

Chemistry Department,

M.V. Lomonosov Moscow State University,

Moscow, Russia

M. P. Kirpichnikov

Faculty of Biology Department,

M.V. Lomonosov Moscow State University,

Moscow, Russia

M. P. Kirpichnikov

M. M. Shemyakin and Yu. A. Ovchinnikov Institute

of Bioorganic Chemistry, Russian Academy of Sciences,

Moscow, Russia

\section{A. R. Khokhlov}

Faculty of Physics Department,

M.V. Lomonosov Moscow State University,

Moscow, Russia

\section{A. R. Khokhlov}

A. N. Nesmeyanov Institute of Organoelement Compounds, Russian Academy of Sciences,

Moscow, Russia that may not be readily accessible to the readership. Third, it is a platform to better explain why the Journal Editorial leadership was enthusiastic about the science and its relationship to the Society on NeuroImmune Pharmacology strategic goals. All are brought to bear in this issue of the Journal of Neuroimmune Pharmacology. The issue includes articles presented at a recent joint US-Russian workshop entitled, "Health in the 21st Century: Nanomedicine and Self-Organization of Biological Systems" held at M.V. Lomonosov Moscow State University (MSU), Moscow, Russia, December 10-11, 2007. The conjoint meeting was organized through the Departments of Biology, Chemistry, and Physics, MSU and by the Center for Drug Delivery and Nanomedicine and Department of Pharmacology and Experimental Neuroscience, University of Nebraska Medical Center (Omaha, NE). The speakers included established internationally regarded scientists from these institutions as well as graduate students and faculties at MSU. In addition to selected papers by workshop contributors, we have included several papers closely aligned to the theme of nanomedicine and nanopharmacology of the central nervous system in order to provide a biological anchor for this research. We understand that such works are new to many but hope that its organization and interdisciplinary approaches will appeal to this audience. All together, it is our hope that, by gathering basic and clinical scientists with the common interest of using nanotechnology in the delivery of therapeutic agents with a focus on nanopharmacology and complex supramolecular biological assembly, the papers included will provide a platform for thought, discussion, and future translational research.

Keywords nanomedicine - neurodegenerative disorders . proteins · glioblastoma · Pluronic P85 .

voltage-gated potassium channels $\cdot$ potassium ion channels 
The issue is led with a discussion of how protein misfolding and aggregation are common threads for neurodegenerative disorders such as Parkinson's disease (Yu and Lyubchenko 2008). Despite the importance of protein misfolding and abnormal protein-protein interactions, factors that lead to aggregation and subsequent disease are poorly understood. Advances in this area of research are highlighted in this article and include single-molecule probing techniques that serve to characterize alpha-synuclein dimers using atomic force microscopy. It is suggested that understanding the molecular mechanisms of protein aggregation during neurodegenerative processes will facilitate studies focused on prevention of protein misfolding and related neuropathologies.

A different aspect of protein self-assembly was considered in the manuscript by Bogush et al. (2008). The investigators describe the use of a combination of electrospinning for physicochemical and molecular modeling used to research relationships between structure and mechanical properties of silk fiber. This study mimics the complete process of spider silk assembly and reports productions of new biomaterials that may be used furthering future biomedical applications for controlled drug release or tissue engineering.

Chekhonin et al. (2008) focus their report on using antibodies specific to glial fibrillary acidic protein (GFAP) and antigen of abluminal membrane of endotheliocytes predominantly expressed in glioblastoma microvessels as a strategy for in vivo targeting of glioblastoma. Based on results presented, the monoclonal antibodies to GFAP can be used as vectors for the delivery of diagnostic and pharmacological agents to high-grade brain tumors. Development of this strategy would open new clinical perspectives for glioblastoma diagnostics and therapy.

Zhang et al. (2008) investigate interactions of a synthetic amphiphilic block copolymer Pluronic P85 in the bloodbrain barrier (BBB). This block copolymer is among the most potent inhibitors of the BBB Pgp efflux system. It is capable of enhancing systemic delivery to the brain of Pgp-dependent drugs, which otherwise are excluded from the central nervous system (CNS) due to insufficient BBB permeability. The data on interactions of the block copolymer with amino acid transport systems are presented and discussed in relation to safety of Pluronic formulations for the BBB.

An interesting hypothesis about the interrelationship of the potassium ion transport and neuroinflammatory responses during human immunodeficiency virus (HIV-1) infection is advanced by the work of Gendelman et al. (2008). They demonstrate using laboratory and animal models of HIV-1 encephalitis that the monocyte chemotactic protein-1 affects the monocyte-macrophage transmigration across the BBB through regulation of potassium voltage-gated channels. This leads to outward $\mathrm{K}^{+}$currents that affect cell shape and mobility and results in increased transmigration across the BBB. This work is also the first described that uses coregistration with single-photon emission computed tomography with standard computed tomography bioimaging to assess the dynamics of monocyte-macrophage migration across the blood-brain barriers and the means to inhibit this pathological process. Altogether, they propose that the potassium ion channels are potential targets for novel therapeutic strategy for neuroAIDS.

The importance of voltage-gated potassium channels in HIV-1 disease is further highlighted by the review by Keblesh et al. (2008). This work reviews the importance of such channels in critical cell functions that are linked to glial biology and neurodegenerative processes linked to neuroAIDS.

The role of the potassium ion channels in cell function is further discussed in a review by Pischalnikova and Sokolova (2008). Because of their critical role in the CNS, all ion channels are targets for multiple pharmacologically active compounds. The authors present the recent data about domain organization of eukaryotic potassium voltage-gated ion channels and discuss the interaction between the domains and the corresponding conformational changes upon activation of the channel. This theme is continued in a study by Nekrasova et al. (2008), which reports on a new bacterial cell-based system, suitable for study of interactions between ligands and ligand-binding sites of eukaryotic Kv1.3 and Kv1.1 channels. This new system may be useful for screening of ligands to membrane-embedded pharmaceutical targets.

In conclusion, the articles in this issue discuss several major problems in nanomedicine, pharmacology, and immunology of the CNS. Therefore, we believe that the present issue will be of considerable interest to academic, government, and industrial scientists and graduate students willing to learn about this fascinating field that is continuously growing. In addition to purely scientific questions, this issue also exposes the new possibilities of scientific cooperation between the USA and Russia, which could involve exchange of ideas and collaborations in the areas where each side has complementary or mutually shared strengths.

\section{References}

Bogush VG, Sokolova OS, Davydova LI, Klinov DV, Sidoruk KV, Esipova NG, Neretina TV, Orchanskyi IA, Makeev VY, Tumanyan VG, Shaitan KV, Debabov VG, Kirpichnikov MP (2008) A novel model system for design of biomaterials based on recombinant analogs of spider silk proteins. J Neuroimmune Pharmacol doi:10.1007/s11481-008-9129-Z

Chekhonin VP, Baklaushev VP, Yusubalieva GM, Gurina OI (2008) Targeted transport of (125)I-labeled antibody to GFAP and AMVB1 in an experimental rat model of C6 glioma. J Neuroimmune Pharmacol doi:10.1007/s11481-008-9123-5

Gendelman HE, Ding S, Gong N, Liu J, Ramirez SH, Persidsky Y, Wang T, Volsky DJ, Xiong H (2008) Monocyte chemotactic protein-1 
regulates voltage-gated $\mathrm{k}+$ channels and macrophage transmigration. J Neuroimmune Pharmacol doi:10.1007/s11481-008-9135-1

Keblesh J, Hu D, Xiong H (2008) Voltage-gated potassium channels in human immunodeficiency virus type-1 (HIV-1)-associated neurocognitive disorders. J Neuroimmune Pharmacol doi:10.1007/ s11481-008-9106-6

Nekrasova OV, Ignatova AA, Nazarova AI, Feofanov AV, Korolkova YV, Boldyreva EF, Tagvei AI, Grishin EV, Arseniev AS, Kirpichnikov MP (2008) Recombinant Kv channels at the membrane of Escherichia coli bind specifically agitoxin2. J Neuroimmune Pharmacol doi:10.1007/s11481-008-9116-4
Pischalnikova AV, Sokolova OS (2008) The domain and conformational organization in potassium voltage-gated ion channels. $\mathrm{J}$ Neuroimmune Pharmacol doi:10.1007/s11481-008-9130-6

Yu J, Lyubchenko YL (2008) Early stages for Parkinson's development: alpha-synuclein misfolding and aggregation. J Neuroimmune Pharmacol doi:10.1007/s11481-008-9115-5

Zhang X, Alakhova DY, Batrakova EV, Li S, Yang Z, Li Y, Kabanov AV (2008) Effect of Pluronic P85 on amino acid transport in bovine brain microvessel endothelial cells. J Neuroimmune Pharmacol doi:10.1007/s11481-0089119-1 